\title{
ADSORÇÃO DO CORANTE VERMELHO PROCION POR NANOTUBOS DE CARBONO FUNCIONALIZADOS
}

\author{
J. C. SLAVIERO ${ }^{1}$, R. CHAVES ${ }^{1}$, E. CHAVES PERES ${ }^{1}$ e G. L. DOTTO ${ }^{1}$ \\ ${ }^{1}$ Universidade Federal de Santa Maria, Departamento de Engenharia Química \\ E-mail para contato: jenifer.slaviero@hotmail.com
}

\begin{abstract}
RESUMO - O objetivo desse trabalho foi avaliar o desempenho de nanotubos de carbono, funcionalizados com $\mathrm{COOH}-\mathrm{e} \mathrm{OH}-$, como adsorventes para a remoção do corante vermelho procion de águas contaminadas. O estudo mostrou que, quanto maior a quantidade de adsorvente empregada, mais eficiente é a remoção do corante, alcançando $99,8 \%$ de rendimento com OH- e 99,5\% com COOH-. A capacidade de adsorção foi influenciada por todos os efeitos testados, sendo que a condição mais favorável ao processo foi à $55^{\circ} \mathrm{C}$ e $\mathrm{pH}$ igual a 2 e 4, para os adsorventes funcionalizados com $\mathrm{COOH}-\mathrm{e} \mathrm{OH}-$, respectivamente. A quantidade máxima atingida sob essas condições, foi de $213,5 \mathrm{mg} . \mathrm{g}^{-1}$ para o nanotubo funcionalizado com $\mathrm{COOH}-$, e $197,7 \mathrm{mg}_{\mathrm{g}} \mathrm{g}^{-1}$ para o funcionalizado com $\mathrm{OH}-$. O modelo que melhor representou os parâmetros cinéticos foi o de Elovich e o que melhor descreveu as isotermas de adsorção foi o modelo de Langmuir.
\end{abstract}

\section{INTRODUÇÃO}

A baixa qualidade das águas naturais é considerada um dos maiores problemas ambientais da atualidade. Grande parcela da contaminação ambiental provém da poluição da água por diferentes substâncias, orgânicas ou inorgânicas. $O$ desenvolvimento de tecnologias para o tratamento de águas contaminadas tem aumentado gradualmente, devido a maior rigidez das legislações ambientais. Corantes são uma das substâncias com maior presença nos efluentes industriais. Quando não são devidamente tratados, acarretam perigoso efeito ao meio ambiente, causando inúmeros problemas de saúde aos animais, plantas e seres humanos que utilizam a água contaminada para diferente fins. De acordo com Gauratini e Zanoni (1999), estima-se que 15 a $20 \%$ da produção mundial de corantes é perdida para o meio ambiente durante a sua síntese, processamento ou aplicação, o que é um fator preocupante visto que cerca de um milhão de toneladas é dispersa no ambiente anualmente. Entre os diversos métodos físicos, químicos e biológicos de tratamento, como coagulação, precipitação química, adsorção, extração por solvente, osmose reversa, a adsorção é alternativa mais empregada por apresentar maior efetividade, principalmente na remoção de corantes do efluente, devido à alta solubilidade do corante em água. Segundo Vuono et al. (2016), nanotubos de carbono ou NCTs funcionalizados estão sendo altamente estudados como adsorventes, devido aos amplos poros, grande área superficial, baixa massa específica e alta interação com várias substâncias químicas. Para aumentar a interação com outras superfícies, se faz necessário o processo de funcionalização, que se trata de um processo químico baseado na inserção de grupos funcionais, como hidroxila, carboxila e carbonila, nas paredes do material, mudando quimicamente a superfície do mesmo. Essas características, no caso da remoção de corantes, originam ligações entre as moléculas do corante e os nanotubos 
funcionalizados, promovendo maior intensidade na adsorção. Este trabalho tem como objetivo estudar a remoção do corante vermelho procion de águas contaminadas, através da adsorção por nanotubos de carbono funcionalizados com $\mathrm{COOH}-\mathrm{e} \mathrm{OH}-$.

\section{MATERIAIS E MÉTODOS}

\subsection{Materiais}

Os nanotubos de carbono funcionalizados com $\mathrm{COOH}-\mathrm{e} \mathrm{OH}$ - foram fornecidos pela empresa Nanostructured \& Amorphous Materials, Inc. O adsorvente apresenta 10 a $30 \mathrm{~nm}$ de diâmetro externo e 5 a $10 \mathrm{~nm}$ de diâmetro interno, massa específica de $2,1 \mathrm{~g} / \mathrm{cm}^{3}$ e pureza de 90\%. O teor de COOH- e OH- é de 1,47-1,63 wt\% (p/p) e 2,36-2,60 wt\% (p/p), respectivamente. O corante utilizado foi o Procion Vermelho MX-5B, com massa molecular de $615,34 \mathrm{~g} / \mathrm{mol}$.

\subsection{Ensaios de adsorção}

Os experimentos foram aplicados igualmente para ambos adsorventes. Empregou-se diferentes massas de adsorvente: 50, 100, 250, 500, 1000, $1500 \mathrm{mg}$, cada quantidade foi colocada em contato com uma solução de $50 \mathrm{~mL}$ do corante vermelho procion de concentração $50 \mathrm{mg} \cdot \mathrm{L}^{-1}$. Todas as soluções foram agitadas durante duas horas e então filtradas. Para avaliar o efeito do $\mathrm{pH}$, a massa de cada adsorvente foi mantida fixa a $50 \mathrm{mg}$. Utilizaram-se 12 soluções de $50 \mathrm{~mL}$ com a mesma concentração de corante, $50 \mathrm{mg} \cdot \mathrm{L}^{-1}$, cada dupla foi ajustada para um $\mathrm{pH}(2,4,6,8,10$ e 12). Ambos os adsorventes empregados foram colocados em contado com a solução em um pH diferente, estas foram agitadas por duas horas e, então, filtradas. Em relação ao tempo de agitação, foi feita a analise cinética, considerando os tempos de 4 a 240 min. A massa de adsorvente e a concentração de corante foram mantidas constantes, $50 \mathrm{mg}$ e $100 \mathrm{mg} \cdot \mathrm{L}^{-1}$, respectivamente. Após a massa prevista de cada adsorvente ser colocada em contato com uma solução de $50 \mathrm{~mL}$ do corante, estas foram submetidas aos tempos de agitação citados anteriormente e, então, filtradas. A influência da temperatura $\left(25,35,45,55^{\circ} \mathrm{C}\right)$ foi avaliada para 3 concentrações diferentes: 50,200 e 500 $\mathrm{mg} \cdot \mathrm{L}^{-1}$. A massa de ambos adsorventes foi mantida fixa em $50 \mathrm{mg}$, após ser colocada em contato com cada concentração, o processo de agitação teve duração de 2 horas, para todas as temperaturas consideradas. As concentrações foram determinadas por espectroscopia no UVvisível para o comprimento de onda de 559nm.

\subsection{Metodologia de cálculo}

Para o ajuste dos dados experimentais, aplicaram-se três modelos para a análise cinética, pseudo-primeira ordem, pseudo-segunda ordem e Elovich, equações (1), (2) e (3) respectivamente:

$$
\begin{aligned}
& \mathrm{q}_{\mathrm{t}}=\mathrm{q}_{\mathrm{e}}\left(1-\mathrm{e}^{\left.-\mathrm{k}_{\mathrm{e}} \mathrm{t}\right)}\right. \\
& q_{t}=\frac{t}{\frac{1}{K_{2} q_{\mathrm{e}}{ }^{2}}+\frac{t}{q_{\mathrm{e}}}}
\end{aligned}
$$


$\mathrm{q}_{\mathrm{t}}=\frac{1}{\beta} \ln (1+\alpha \beta . t)$

Onde $K_{1}$ é a constante da taxa de adsorção de pseudo-primeira ordem, $\mathrm{q}_{\mathrm{e}}$ e $\mathrm{q}_{\mathrm{t}}$ são as quantidades adsorvidas por grama de adsorvente no equilíbrio e no tempo $t$, respectivamente. $K_{2}$ é a constante da taxa de adsorção de pseudo-segunda ordem. Para a equação de Elovich, $\alpha$ é taxa de adsorção inicial e $\beta$ é a constante de dessorção.

Foram usados dois modelos para o ajuste das isotermas de adsorção, o modelo de Langmuir e de Freundlich, equações 4 e 5, respectivamente:

$q_{\mathrm{B}}=\frac{q_{\max } \cdot K_{L} \cdot C_{\mathrm{B}}}{1+K_{L} C_{\theta}}$

$\mathrm{q}_{\mathrm{e}}=\mathrm{K}_{\mathrm{F}} \cdot \mathrm{C}_{\mathrm{e}}^{\frac{1}{\mathrm{n}}}$

Onde qe é a quantidade adsorvida por massa de adsorvente no equilíbrio, $\mathrm{Ce}$, a concentração

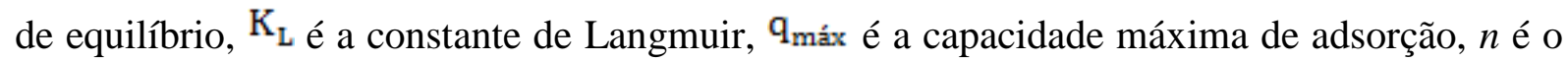
parâmetro de Freundlich e $\mathrm{K}_{\mathrm{F}}$ é a constante de Freundlich.

\section{RESULTADOS E DISCUSSÃO}

\subsection{Efeito da dosagem de adsorvente}

Após análise das amostras obtidas por espectrometria, foi possível avaliar a influência da dosagem do adsorvente no processo de adsorção, como mostra a Figura 1. Pode-se observar que a eficiência de adsorção elevou-se juntamente com o aumento da massa de ambos adsorventes avaliados, sendo o maior rendimento alcançado para a maior massa considerada, $1500 \mathrm{mg}$. Os nanotubos de carbono funcionalizados com $\mathrm{OH}-$ e funcionalizados com COOH- alcançaram um percentual de remoção de 99,8\% e 99,5\%, respectivamente.

Figura 1 - Efeito da dosagem dos adsorventes no processo de adsorção

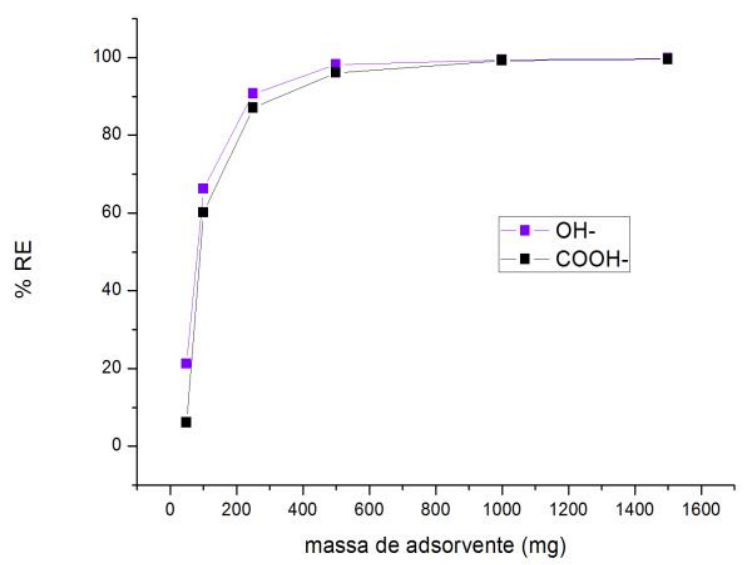




\subsection{Efeito do pH}

O efeito do pH da solução na capacidade de adsorção se mostrou diferente para os dois adsorventes utilizados. A amostra que foi preparada a partir de uma solução a pH 4 demonstrou os melhores resultados para os nanotubos funcionalizado com $\mathrm{OH}$ - conforme a Figura 2, já para o funcionalizado com $\mathrm{COOH}-$, o $\mathrm{pH}$ que mais favoreceu o processo de adsorção foi o 2. Entretanto, a capacidade máxima de adsorção foi a mesma para os dois adsorventes funcionalizados, alcançando $49,16 \mathrm{mg} . \mathrm{g}^{-1}$.

Figura 2 - Efeito do pH na adsorção do corante vermelho procion
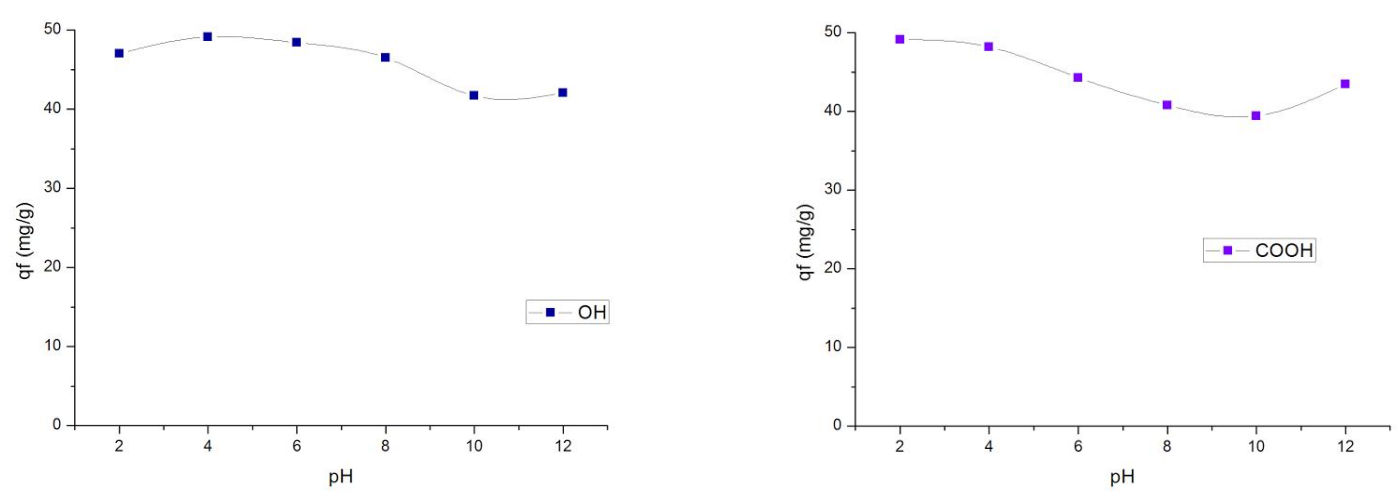

\subsection{Cinética da adsorção}

Na Figura 3, são apresentados os resultados para a análise cinética, com a concentração inicial de $100 \mathrm{mg} \cdot \mathrm{L}^{-1}$.

Figura 3 - Efeito da cinética no processo de adsorção

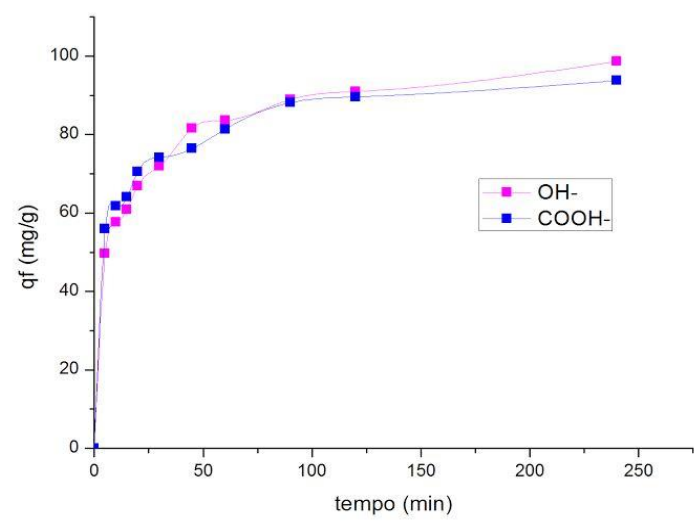

Os nanotubos de carbono funcionalizados com $\mathrm{OH}$ - apresentaram uma quantidade de corante adsorvida superior à do COOH-, sendo a máxima capacidade de adsorção 98,7 mg.g $\mathrm{g}^{-1}$, e 93,8 mg.g ${ }^{-1}$, respectivamente. Observa-se que o após 2 horas, aproximadamente, o sistema 
atingiu o equilíbrio, não ocorrendo mais mudanças significativas na quantidade de substâncias adsorvidas. Os parâmetros cinéticos obtidos de cada modelo são mostrados na Tabela 1:

Tabela 1- Parâmetros cinéticos de adsorção do corante Vermelho Procion

\begin{tabular}{ccc}
\hline Adsorvente & OH & COOH \\
\hline Pseudo-primeira ordem & $\mathrm{C} 0=100 \mathrm{mg} / \mathrm{L}$ & $\mathrm{C} 0=100 \mathrm{mg} / \mathrm{L}$ \\
\hline $\mathrm{q}_{1}(\mathrm{mg} / \mathrm{g})$ & 86,4 & 82,4 \\
$\mathrm{k}_{1}(1 / \mathrm{min})$ & 0,0994 & 0,1485 \\
$\mathrm{R}^{2}$ & 0,8488 & 0,7587 \\
$\mathrm{ARE}(\%)$ & 9,18 & 8,83 \\
\hline Pseudo-segunda ordem & & \\
\hline $\mathrm{q}_{2}(\mathrm{mg} / \mathrm{g})$ & 94,8 & 89,3 \\
$\mathrm{k}_{2}$ x $10^{3}$ (g mg/min) & 1,5677 & 2,5765 \\
$\mathrm{R}^{2}$ & 0,9563 & 0,9276 \\
$\mathrm{ARE}(\%)$ & 4,83 & 5,03 \\
\hline Elovich & & \\
\hline $\mathrm{a}(\mathrm{g} / \mathrm{mg})$ & 0,0740 & 0,0946 \\
$\mathrm{~b}(\mathrm{mg} / \mathrm{g}$ min) & 98,1 & 378,9 \\
$\mathrm{R}^{2}$ & 0,9938 & 0,9918 \\
$\mathrm{ARE}(\%)$ & 1,70 & 1,57 \\
\hline
\end{tabular}

A avaliação dos modelos foi realizada pela comparação dos coeficientes de determinação $\left(\mathrm{R}^{2}\right)$ e erro médio relativo (ARE). Verificou-se que o modelo de Elovich foi o que melhor representou os dados experimentais.

\subsection{Isotermas da adsorção}

Para avaliar o efeito da temperatura na capacidade de adsorção do corante por ambos os adsorventes, foram traçadas as isotermas de adsorção no equilíbrio, para as três concentrações consideradas, como mostra a Figura 4.

Figura 4 - Isotermas de adsorção do corante vermelho procion
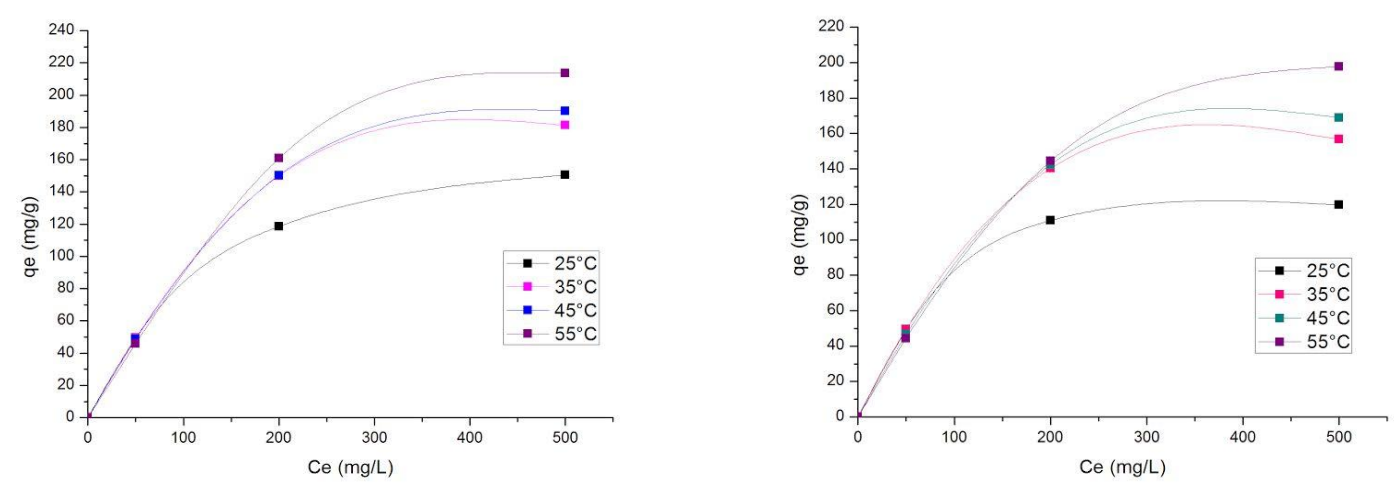

Observa-se que o aumento da temperatura favorece a adsorção. Os melhores resultados foram obtidos a $55^{\circ} \mathrm{C}$. Quanto à capacidade máxima de adsorção, o adsorvente funcionalizado com COOH- obteve melhores resultados, alcançando $213,5 \mathrm{mg} \cdot \mathrm{g}^{-1}$, enquanto o funcionalizado 
com OH- atingiu 197,7 mg. $\mathrm{g}^{-1}$ As isotermas foram descritas de acordo com os modelos de Langmuir e Freundlich como mostra a Tabela 2.

Tabela 2 - Parâmetros das isotermas de adsorção do corante vermelho procion

\begin{tabular}{ccccccccc}
\hline Adsorvente & \multicolumn{3}{c}{ OH- } & \multicolumn{5}{c}{ COOH- } \\
\hline Freundlich & $\mathbf{2 9 8 K}$ & $\mathbf{3 0 8 K}$ & $\mathbf{3 1 8 K}$ & $\mathbf{3 2 8 K}$ & $\mathbf{2 9 8 K}$ & $\mathbf{3 0 8 K}$ & $\mathbf{3 1 8 K}$ & $\mathbf{3 2 8 K}$ \\
\hline $\mathrm{kF}(\mathrm{mg} / \mathrm{g})(\mathrm{mg} / \mathrm{L})-^{1 / \mathrm{nf}}$ & 74,1 & 70,2 & 55,5 & 45,4 & 61,9 & 70,1 & 64,3 & 57,6 \\
$1 / \mathrm{nF}$ & 0,082 & 0,149 & 0,207 & 0,266 & 0,149 & 0,173 & 0,198 & 0,240 \\
$\mathrm{R}^{2}$ & 0,823 & 0,951 & 0,934 & 0,957 & 0,978 & 0,973 & 0,971 & 0,942 \\
$\mathrm{ARE}(\%)$ & 12,41 & 11,73 & 15,66 & 15,96 & 6,24 & 10,16 & 11,33 & 19,11 \\
\hline Langmuir & $\mathbf{2 9 8 K}$ & $\mathbf{3 0 8 K}$ & $\mathbf{3 1 8 K}$ & $\mathbf{3 2 8 K}$ & $\mathbf{2 9 8 K}$ & $\mathbf{3 0 8 K}$ & $\mathbf{3 1 8 K}$ & $\mathbf{3 2 8 K}$ \\
\hline $\mathrm{qm}(\mathrm{mg} / \mathrm{g})$ & 116,0 & 152,4 & 171,6 & 195,2 & 132,6 & 170,0 & 179,4 & 206,8 \\
$\mathrm{~kL}(\mathrm{~L} / \mathrm{mg})$ & 1,85 & 0,53 & 0,13 & 0,07 & 0,61 & 0,42 & 0,25 & 0,12 \\
$\mathrm{R}^{2}$ & 0,919 & 0,994 & 0,986 & 0,969 & 0,942 & 0,983 & 0,984 & 0,955 \\
$\mathrm{ARE}(\%)$ & 9,03 & 2,62 & 6,70 & 11,69 & 8,95 & 5,78 & 3,92 & 15,82 \\
\hline
\end{tabular}

Pelos resultados obtidos nota-se que o modelo de Langmuir foi mais adequado para representar os dados experimentais, já que, apresentou os menores erros médios relativos (ARE) e os maiores coeficientes de determinação $\left(\mathrm{R}^{2}\right)$ em comparação com o modelo de Freundlich. As capacidades máximas de adsorção, de acordo com os ajustes do modelo de Langmuir, foram de 195,2 mg. $\mathrm{g}^{-1}$ para o adsorvente funcionalizado com $\mathrm{OH}-$, e de 206,8 mg. $\mathrm{g}^{-1}$ para o funcionalizado $\mathrm{COOH}-$. De acordo com os resultados, é possível observar que a capacidade de adsorção máxima através dos nanotubos funcionalizados com $\mathrm{COOH}^{-}$é cerca de $6 \%$ maior que o adsorvente funcionalizado com $\mathrm{OH}^{-}$.

\section{CONCLUSÃO}

Através dos experimentos realizados, determinou-se que a remoção do corante vermelho procion por adsorção é favorecida para soluções ácidas, com alta concentração de adsorvente e na temperatura de $55^{\circ} \mathrm{C}$. No equilíbrio, as quantidades máximas de adsorção atingiram 213,5 mg. $\mathrm{g}^{-1}$ para o $\mathrm{COOH}-$ e $197,7 \mathrm{mg}^{-1} \mathrm{~g}^{-1}$ para o $\mathrm{OH}-$. Os nanotubos de carbono funcionalizados com $\mathrm{OH}-\mathrm{e} \mathrm{COOH}-$ se mostraram bons adsorventes por atingirem altas capacidades de adsorção e altos percentuais de remoção. Portanto, apresentam uma válida alternativa para a remoção do corante vermelho procion de efluentes líquidos.

\section{REFERENCIAS}

Guaratini, C. C. I.; Zanoni, V. B. Corantes Têxteis. Química Nova, p. 71-78, 1999.

Vuono, D.; Catizzone, E.; Aloise, A.; Policicchio, A.; Agostino, R. G.; Migliori, M.; Giordano, G. Modelling of adsorption of textile dyes over multi-walled carbon nanotubes: Equilibrium and kinetic. Chinese Journal of Chemical Engineering, 2016. 